\title{
User-Friendly: Anthropomorphic Devices and Mechanical Behaviour in Japan
}

\author{
Michael Shea \\ Culture, Materials and Design, Department of Anthropology, University College London, UK \\ Email: michael.shea.10@ucl.ac.uk
}

Received September $16^{\text {th }}, 2013$; revised October $18^{\text {th }}$, 2013; accepted November $15^{\text {th }}, 2013$

\begin{abstract}
Copyright (c) 2014 Michael Shea. This is an open access article distributed under the Creative Commons Attribution License, which permits unrestricted use, distribution, and reproduction in any medium, provided the original work is properly cited. In accordance of the Creative Commons Attribution License all Copyrights (c) 2014 are reserved for SCIRP and the owner of the intellectual property Michael Shea. All Copyright (C) 2014 are guarded by law and by SCIRP as a guardian.
\end{abstract}

\begin{abstract}
Anthropomorphic avatars and disembodied voices have become part of everyday life in Japan. From the animated characters that bow after you complete a transaction at an automated teller machine to the phenomenal proliferation of consumer goods bearing cute faces. There is a discernable growing tendency to anthropomorphize machines. These anthropomorphic devices stand in contrast with the somewhat automated nature of many human interactions. Particularly in the behavior required of employees that work in customer service roles, which calls to mind the demand that workers must often behave as machines from which the notion of a robot originates. Based on research conducted at the National Museum of Emerging Science and Innovation in Tokyo, examples not only of how friendliness can be mechanically produced but also of new devices being imbued with functions to demonstrate their friendliness will be critically examined.
\end{abstract}

Keywords: Japan; Robotics; Anthropomorphism; Technology; Museum Education

\section{Introduction}

At five minutes before five o'clock the museum at Miraikan's imminent closing is indicated by a recorded loudspeaker announcement. It plays on loop, thanking visitors in English, Japanese, Chinese and Korean. This is followed by an instrumental version of Auld Lang Syne. This song is often used in bars, restaurants and izakaya to indicate closing time. I comment on the strangeness of the song being used in this manner as in the United Kingdom it is solely associated with New Year's Eve celebrations, to a volunteer who does not recognize the title however on hearing the tune exclaims "Oh! The going home song!" Another volunteer confides in me that the leaving music is his favorite sound "I love it when the music starts! Means it's time to go home..." Once this music begins the staff begin to gently encourage the visitors to leave, though never directly. This is achieved by standing in rows at a right angle to the exit and thanking and bowing to visitors as they pass. Unlike the staff the volunteers are not obliged to wait until the last visitors leave however they usually do. When I bow I look like an idiot, but I attempt to do so nonetheless. The museum is usually empty of customers within ten minutes of closing. The gentleness of the closing procedures, so as never to directly confront a visitor with an order to leave are reminiscent of the kind of tactics used in upscale retail stores. The level of politeness and reminds me of time spent working in a department store in England.

A key function of the "Science Communicators" and volunteers at Miraikan is to walk among the museum exhibits and to give visitors information as they request it. The most common question visitors ask is "where is the toilet?" however due to the nature of the exhibits the next visitor might ask for an explanation of 3D printing or quantum computing. SC's and volunteers are therefore expected to be able to give answer such questions in a friendly and approachable manner. This kind of informal guidance is the volunteers' main role at the museum. Being unpaid they are usually required to do little but stand on the exhibit floor and be available to talk with visitors. I found that this freedom to wander and talk to strangers made acting as a volunteer was quite conducive to participant observation. When the museum is quiet it could become a little awkward as the staff and volunteers patrolling the exhibition space outnumbered visitors. There is a skill in being present and available to visitors without being overbearing. The patterns of walking slowly and maintaining open gestures without too much eye contact are familiar to anyone who has ever worked in retail or other similar service roles. By a chance encounter with a researcher at the Advanced Telecommunications Research Institute I found a name for this sort of behavior: friendly patrolling.

\section{Friendly Patrolling}

On a visit to the ATR laboratory I witnessed a public demonstration of "Robovie II" to a group of visiting schoolchildren. Robovie is an interactive humanoid robot, characterized by humanlike physical expressions and various sensors. It recognizes sight, sound and touch. Robovie is $120 \mathrm{~cm}$ high and 40 $\mathrm{cm}$ wide, with eyes, two arms and a metal torso on wheels. It has a decidedly retro appearance roughly resembling Robby the Robot from Forbidden Planet. I had encountered this model of 
Robovie before at a different laboratory, in half working order, where I was invited to grab the robot's arm in order to demonstrate its touch sensors. Grabbing its forearm caused it to jerk its head towards me as an acknowledgement of the touch in quite an unsettling way. In this demonstration the children were taking it in turns to walk past Robovie and be greeted, thereby showing the robot's spatial awareness as it must navigate around the person. It approaches, says hello, the young girl says hello back, and then giggles and runs away. There is a monitor nearby with a graphic representation of how Robovie is navigating the controlled space where the procession of schoolchildren is being greeted. I listened to the researcher explaining the images on the monitor for a little while and later read some of ATR's material describing past experiments using Robovie, including one titled "Friendly Patrolling" in which the robot was used to imitate the behavior of security staff at a shopping mall. The intention was to create a model of how staff must patrol an area in a visible, authoritative and yet approachable way. This was achieved by recording the movements of security staff at a mall and then having Robovie repeat the same walking patterns. The model for friendly patrolling was created based on recording the movements of experienced security guards at a shopping mall using positional and video data.

Friendly patrolling is described as follows: "In daily life, security guards police officers and sales clerks roam around environments and nonverbally present friendly behavior so that people feel comfortable talking to them. We modeled the behavior of human experts during friendly patrolling, which we defined as a roaming behavior that nonverbally presents a friendly attitude, to encourage people to talk to such professionals" (Hayashi et al., 2011: p. 1). The authors go on to argue that in order for robots to operate successfully in such roles they must learn to conform to expected behaviors: "friendly patrolling is an expected behavior for natural encounters with people in such services as staff in shops, staff at train and bus stations and guards and police officers in public environments in some countries (hopefully in most countries)" (Hayashi et al., 2011: p. 2). The acknowledgement that the expected behavior of service and security staff might vary from country to country is important as this is arguably the case.

Obvious examples of ways in which service staff are expected to behave differently in Japan compared to elsewhere include bowing and other gestures as well as the use of son keigo, respectful language used exclusively when speaking to superiors or customers in a professional environment. The experiment used thirty-nine participants and was conducted in a shopping mall. Two parameters were controlled in the study: "the speed with which the robot moved when near a pedestrian and the distance kept. The researchers acknowledged that these parameters were specific to the robot they used and perhaps to Japanese people” (Hayashi et al., 2011: p. 8), again raising the possibility of the cultural specificity of these patterns of behavior. The research findings rather unnervingly mention the choice of modeling the behavior of security guards was made on the basis that in the future robots will be used for surveillance and security in public spaces. Concluding: "will these robots simply patrol and intimidate people? We believe that such robots in the future will be used in friendly and socially acceptable manners. In other words, these robots need a function to show their friendliness" (Hayashi et al., 2011: p. 7). This summary gets to the heart of what friendly patrolling is: a function to show friendliness. I would argue that many aspects of this mode of behavior are culturally specific and that it must be learned, as with any social convention. The attempt to model and emulate such gestures with a machine serves to highlight the mechanical nature of these patterns of behavior. Put simply, service staff must necessarily become like a robot.

Henri Bergson famously recorded that "The attitudes, gestures, and movements of the human body are laughable in exact proportion as that body reminds us of a mere machine" (1956: p. 72). This certainly was my experience when I tried to learn to bow correctly. This was during volunteer training day ahead of beginning my research at the museum. This included a brief training session in polite behavior, how to gesture and bow correctly to visitors, as well as learning some son keigo phrases. I was amused when the woman leading the politeness training explains how in England all people leave school with this level of etiquette training. It occurs to me that many paid jobs do not give this level of introductory training, as I repeatedly (and hopelessly) practice bowing facing a fellow new volunteer doing the same. To a foreigner, the sight of people in service positions bowing repeatedly can be at times both humorous and unsettling. In Sofia Coppola's "Lost in Translation” there is a humorous scene where the protagonist walks in and out of a Tokyo hotel lobby repeatedly, causing the staff bow repeatedly in quick succession. Bowing provides a good example of social gesture that causes people to act like machines. By jerking forwards in a controlled fashion, the onlooker is presented with a deliberate act of humility. One that is formal, regimented and difficult to misinterpret. Luckily Miraikan is only a semi-formal service environment so bowing is less frequent and less intensely regimented than it would be in say a hotel or restaurant. The staff that act as greeters and work on the reception desks (exclusively young women) bow more often and with the most skill of all the staff and volunteers. They also have a more complex uniform than the SC's and volunteers, consisting of a purple vest and underneath it, an inferably Star Trek inspired grey skinny jump suit. Science Communicators dress casually and must only wear a white vest to identify themselves as staff. Volunteers have the same vest, but in garish orange, making it considerably more conspicuous.

Bowing can be humorous as it makes a person appear mechanical, by repeating unnatural movements like the figures on a cuckoo clock. Arguably the purpose of this and other formal gestures is a kind of "othering" in order to demonstrate the distinction between service staff and those that they serve. Friendly patrolling, is interesting in this regard because although staff and volunteers are acting in a service role, in order to engage with visitors they must be approachable. Therefore many of the characteristics of friendly patrolling as it is performed at Miraikan is to counter the rigid distinction between staff and customer. Volunteers in particular problematize this distinction as all are unpaid and therefore tend to reasonably affluent, wandering the exhibits in much the same way as a visitor would and necessarily acting quite casually in order to facilitate friendly conversations with visitors. In short, friendly patrolling encompasses a paradox of being approachable yet distant, as volunteers straddle the divide between service staff and customer.

If service staff must behave as "other" than perhaps it is unsurprising that there has long been a dream to have these roles provided by robots. As Arthur Harkins succinctly puts it "Why have a machine with a mind of its own who can say 'No!'? People in general prefer the appliance robot” (1983: p. 29). In 
Tokyo already there are anthropomorphized devices all around, as soon as you begin to look for them. On the subway the doors speak (incidentally, automated train announcements are an excellent way to learn son keigo phrases), animated characters bow to you at cash points when you complete a transaction, at many bars tablet computers have taken the place of waiters, the food is served by humans but the communication between the customers and the staff happens almost exclusively via the computer. Mark Gilson is one of a multitude of cultural commentators to emphasize the tech friendliness of Japanese culture, noting the anthropomorphic quality of the branding of many consumer electronics, i.e. "Game Boy” and "Discman” (1998: p. 369). Service functions have been increasingly automated for a long time, evident in the widespread use of vending machines to place orders in everywhere from restaurants to hairdressers. Just as volunteers at the museum have to perform their friendliness in order to make human connections, there are many situations where a highly formalized, even automated interaction is preferable, hence the enthusiasm for automation and the idea of a service robot. In an interview with Shuichi Nishio, a researcher at Intelligent Robotics and Communication Laboratories, Advanced Telecommunications Research Institute International the practical application of tele-operated humanoid robots were described as follows: "As a new communication medium that can transmit not only conversational information but human presence. Also, the semi-teleoperational nature of the androids allows various applications that require professional knowledge occasionally but for the most time routine responses are only necessary, such as a sales receptionist." The choice of receptionist as a role that could be filled by an android is interesting as it hints at nature of these kinds of service positions.

One section of the Asimo robot demonstrations at Miraikan is the roboto no shokai (robot introduction) where Asimo tells the audience about another robot and explains its purpose. The audience is shown footage of the robot being described and are asked what they think it is and could be used for before Asimo gives the answers. The robot that is introduced varies from show to show, however the one that tends to get the most powerful reaction from the audience is the female android HRP-4C. Unlike Asimo the android has been given a convincing (and attractive) human face, although its movements immediately make the onlooker realize it is a machine. Lacking in expressions or fluidity of movement, the backing dancers serve to underline its relative stiffness. Impressive nonetheless, and usually elicits genuine surprise and awe from the crowd. It was created by the National Institute of Advanced Industrial Science and Technology and unveiled in 2009 for potential use in the "entertainment" industry. The accompanying press release described how it's specifications were chosen: "Looks like a human being with a height of $158 \mathrm{~cm}$ and body weight of $43 \mathrm{~kg}$ (including the battery)", and the positions of the joints and dimensions are set to the average values for young Japanese females in the "Japanese Body Dimension Database 1997-98" (AIST 2009). The same press release refers to how earlier models in the series have proven potential for use in "3D" jobs (dirty, dangerous and demanding): "with equipment offering dust-proof and drip-proof performance as well as excellent moving functions." Asimo's introduction does not refer to the potential of HRP-4C instead focusing on the entertainment side. It was usually described as the first android pop star or fashion model in technology news reportage that accompanied its re- lease two years ago. There is a clear gender aspect to the design and intended uses of a pretty female android that was designed based on the measurements of young Japanese women. Asimo provides and interesting contrast being purposefully designed to be gender neutral, though often wrongly identified as male.

The implications of creating a beautiful female robot that is able to fulfill various service and "entertainment" roles is one that has been extensively explored in science fiction writing, beginning with Auguste Villiers de I’Isle-Adam's Tomorrow’s Eve:

"Since our gods and our aspirations are no longer anything but scientific, why shouldn' our loves be so, too? In place of that Eve of the forgotten legend, the legend despised and discredited by Science, I offer you a scientific Eve- the only one, I think, now worthy of those blighted visceral organs which you still-by a kind of sentimentality that you're the first to mock-still call "your hearts". Far from being hostile to the love of men for their wives - who are so necessary to perpetuate the race (at least until a new order of things comes in), I propose to reinforce, insure, and guarantee that love. I will do so with the aid of thousands and thousands of marvelous and completely innocent facsimiles, who will render superfluous all those beautiful but deceptive mistresses ineffective henceforth forever. These new beings will function in a second nature, rendered more perfect by Science, and at the very least their healthful assistance will render less painful the miseries that-say what you will-always attend sooner or later your hypocritical marital lapses" (2001: p. 164).

There is evidence of latent misogyny not only in the design of such androids but in how they are viewed and interpreted. For instance take Chris Carroll's article for National Geographic that describes his experience visiting Actroid-DER, a female android developed by Osaka University and manufactured by Kokoro, at Carnegie Mellon University. Carroll describes the android as follows: "can be rented to serve as a futuristic spokesmodel at corporate events, a role that admittedly does not require great depth of character. But in spite of the \$250,000 spent on her development, she moves with a twitchy gracelessness, and the elasticity of her features lends a slightly demented undertone to her lovely face” (2011: p. 72). He goes on to describe how researchers gave the android a makeover, giving it new clothes and renaming it "Yume" (dream) in order to make it more believably human. The result: "In spite of her outfit-including the long fingerless gloves designed to hide her zombie-stiff hands and the dark lipstick that covers up her inability to ever quite close her mouth-underneath she's the same old Actroid DER. At least now she knows her place” (Carroll, 2011: p. 84). These choice observations tell more about the author's attitudes towards women than they do about the technology itself. The role of receptionist or "spokesmodel” may not necessarily lend itself to being performed by women but the desire to create an artificial woman to perform these tasks is instructive of how these kinds of service roles are viewed.

We have seen how service staff may behave like robots and robots are being designed as ideal replacements for service staff. But what of other less formal roles where friendliness is necessary? Another recent experiment to make use of Robovie II's services was conducted last year with a view to seeing if a robot could be used to provide companionship to the elderly. For 
three months Robovie was placed in an elderly care center to support the elderly through conversation:

“This study used Robovie, which was remotely controlled by an operator from an operations room. A set of conversations and gestures were pre-programmed prior to the deployment based on the recommendations of the staff, and no changes were made during the experiment. First thing in the morning, the robot was moved around the rooms where activities were held to perform other tasks, which included conversation and encouragement to the elderly performing difficult tasks” (Sabelli et al., 2011: p. 38).

The robot is described as a support tool for the staff perhaps somewhat devaluing the importance of social contact between staff and patients themselves. The robot was remotely operated by a non-roboticist, Japanese speaker. The instructions to them included: "a set of attitudes (to provide positive feedback or avoid negative comments; grab the attention of the elderly; follow the direction of the conversation initiated by the elderly) and tasks (greet elderly when entering and leaving the room, call their names, encourage them when performing tasks) ... answer questions and comments while avoiding negative remarks and reinforcing positive statements made by the elderly; call the elderly by their name whenever appropriate, and ask questions of the kind 'what is this?' like children do" (Sabelli et al., 2011: p. 38). It is important to note that Robovie was being remote controlled and therefore arguably the residents were engaged in a dialogue with its controller rather than the machine itself. Whether it is simply a novelty where the user "plays along" for their own amusement or whether these devices are actually able to manipulate the users' emotional state is difficult to gage. The authors would dispute this on the grounds that the residents "believed" that they were communicating with the robot (Sabelli et al., 2011: p. 39). It seems to have taken a significant amount of human labour to facilitate the conversations between Robovie and the elderly, so the staff needn't worry about their jobs for the time being. However, this experiment does highlight how there is a continued desire to see service roles that require a decree of emotional involvement be occupied by robots. There is a movement towards making machines easier to interact with, friendly even, that can be seen in the design of consumer electronics, particularly those intended for the domestic sphere. Household appliances must be userfriendly.

\section{User-Friendly}

Household appliances are often steeped in the language of utility, where terms such as "user-friendly" are used to denote the degree of ergonomic functionality of a given product. But there are various less tangible qualities that can determine an appliance's commercial viability. Aesthetics, novelty, humor, even emotions play a crucial role in the success or failure of a domestic product. In Japan this is all too well understood, where a culture of kawaii, of novel-cuteness, dictates that domestic appliances must not only be functional, they must be attractive. Inge Daniels in her ethnography of engimono (good luck charms) argued against any neat dichotomy between utility and aesthetic value of objects in the domestic sphere by considering how even commercial items such as electronic goods can often "blur the distinction between use and symbolic value" (2003: p. 632). Occhi argues that the contemporary brand mas- cot kyara (characters) owe their existence to a tradition of anthropomorphism in Japanese art that is at least nine hundred years old, giving chojujinbutsu giga (caricature of human-animal characters) as historical examples of this technique. Describing one such artwork: "beginning with the depiction of a Sumo match, the first of its four parts consists of a series of scenes of animals including frogs, rabbits and monkeys engaged in human activities (for example, dancing, swimming, running, shooting bows and arrows)” (2012: p. 117).

In Japan household goods are often anthropomorphized in a cartoonish way in order to increase their aesthetic appeal. This effect is amplified once the anthropomorphized device is capable of socially interacting with its user as in the case of some robotic devices currently under development. Technology for use in the domestic sphere has the potential to shape the spaces it inhabits, even to the extent of impacting on one's social relationships, as was recorded in Church et al.'s discussion of media technology in Australian homes (2010: p. 265). The following is a discussion of such appliances in order to explore their potential for establishing an affective relationship with a user, beyond their functional properties, literally their "userfriendliness".

In the "Innovation and Future" section of Miraikan, where I conducted most of the participant observation for this research, one day I noticed that attached to a board behind a colleague's desk is a pair of eyes, made of LED lights (Figure 1). These occasionally appear to gaze left and right as if taking note of those visitors who walk past. Someone has drawn a face on the board around where the eyes are placed in order to complete this illusion of a face stuck in the wall. Occasionally a member of staff will pull the eyes off of the wall (they are attached with velcro) and attach them to something else in order to demonstrate how they work. A computer monitor, or sometimes the surface of the desk itself is transformed into a face in such a way. I have encountered these eyes before, at the Anzai-Imai laboratory of Keio University, where a lead researcher in utilizing human-like robotic parts, Hirotaka Osawa, explained them to me.

The eye-like devices are made of OLED panels, with motion sensors and, among other applications, they have been used as part of a system Dr Osawa had developed to give appliances faces and arms, so that an appliance might be able to explain

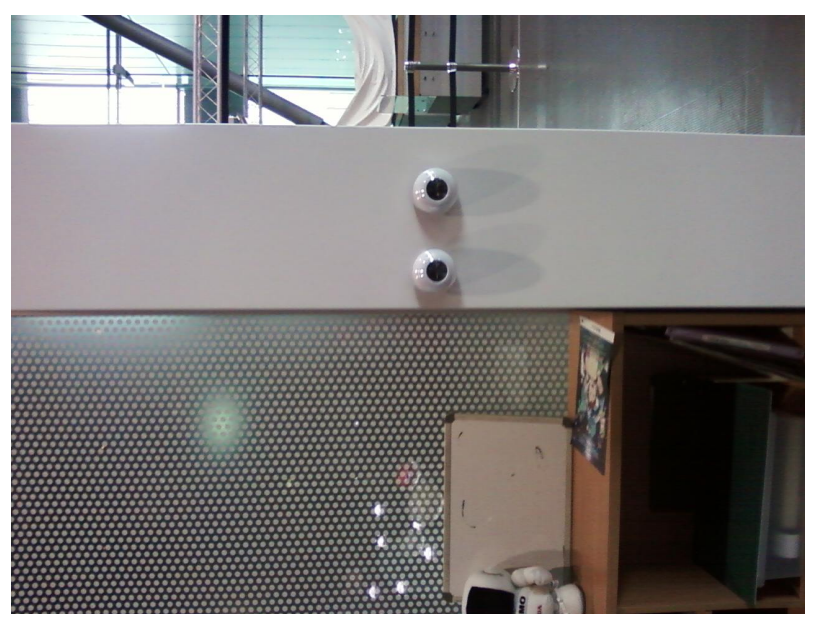

Figure 1.

Robotic eyes on the wall at Miraikan. 
itself and give instructions and feedback to its user. On our first meeting he used a past presentation slideshow as well as the device itself to explain the purpose and potential utility of giving appliances faces and arms. I sat opposite an ordinary vacuum cleaner, but for the addition of a face and arms, as its voice and gestures guided me around its various functions. In order to facilitate better interaction between humans and machines, robotic parts that give a machine a face, voice and the ability to gesture to its user were the primary purpose of developing the system, which I will refer to from now on as the vacuum cleaner gesture robot. It could potentially be used with most appliances. However when I encountered the system it was being used with a vacuum cleaner, as it was in the experiments I will refer to later in this discussion. Osawa describes the system as giving appliances the ability to "explain their functions by themselves" using the coordinated displays, motor, sensor, microcontroller and wireless devices that constitute the face and arms.

The hypothesis underlying Osawa's research is that people interact better with devices if they anthropomorphize them. In his words: "Our proposed humanoid parts redefine the currently ambiguous boundary between 'the ability to perform a task' and 'the ability to act humanly' in a communication robot" (2009: p. 109). Indeed to a child, the definition of a robot can be as simple as any "machine that can talk" (Webb, 1983: p. 5). Under these terms any appliance with the ability to interact with its user socially would fulfil this criterion. There are already examples of this principle in use (human voices giving directions in car GPS systems etc). There are also some particularly Japanese examples, for instance computerized voices and graphics of a bank teller on a cash point ATM screen that says "thank you" and bows as you complete a transaction (Figure 2). There are also functional benefits to giving a machine the ability to speak and gesture, in that it may better convey spatial information. Put simply, a vacuum cleaner that can point to its power button may be easier to use than one that comes with a written diagram. The gesture robot's anthropomorphic features are summarized in an article which argues that it can be easier to use for the elderly in particular: "The gesture robot creates a human-like appearance and assists voice interaction with human-like gestures, emotions, social acts and conceptual metaphors" (2010: p. 179). Emotions are included alongside its

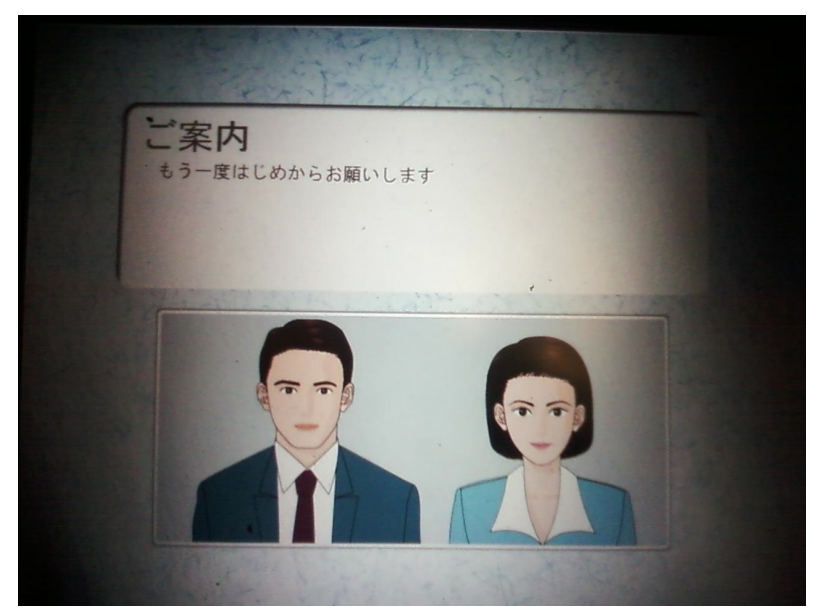

Figure 2.

Automatic teller machine avatars. more tangible abilities.

In a related experiment, where a participant took instructions from a refrigerator fitted with the same eye-like parts about what items in the kitchen to collect and place inside of it. A voice would be heard instructing the participant to retrieve objects and place them in the refrigerator's "stomach". The experiment was then conducted once with the robotic eyes and once without to see what difference anthropomorphism made to the participants' ability to understand the refrigerator's instructions: "To clearly detect participant action, we conducted the experiment by the 'Wizard of Oz' method. In this method, all of the object's motions are watched by the hidden experimenter" (2009: p. 114). The researchers described how the control group, who heard spoken instructions without the addition of robotic eyes gesturing, found it more difficult to interpret the refrigerator's commands: "they were unable to understand who was talking; in other words, they were unable to understand the anthropomorphic representation of words like 'want' or 'my stomach.' In fact, after the final instruction 'Please put them into my stomach,' one participant held an object to his stomach. This action was caused by the participant's inability to see the body image of the refrigerator" (2009: p. 115).

It is hoped that appliances with this kind of social interface may be especially useful for the elderly or physically impaired. This is part of a broader belief within Japanese robotics that the future lies in elderly medical and domestic care, as is well documented in Jennifer Robertson's discussion of the government led initiative "Innovation 25". This was Prime Minister Abe's plan, announced in 2007, for a remaking Japanese society by the year 2025 based around the increasing integration of domestic androids. It sets out a blueprint for accommodating the rapidly aging population through technological innovation such as household robots. Robertson saw in "Innovation 25" an emphasis on "the central role that household robots will play in stabilizing core institutions, like the family" (2007: p. 370). In our discussion Osawa did add that the gesture robot could also be useful for children:

"I think that the gesture robot is good for both children/infants and older people. Acquired knowledge is sometimes lost, however, innate habits are never forgotten. Children/infants have sometimes not have acquired knowledge, but they can respond to anthropomorphic features because they are innately programmed to do so. Older people may forget things they are taught. But if the features have a more anthropomorphic attitude, they are less easy to forget."

Here Osawa is referring to the idea that there are innate human abilities to respond to other's expressions and gestures that mean that those with impaired memories or limited capacity for abstract thought (as in the case of infants) could benefit from having an appliance that can direct them in this manner. Before addressing these claims directly, it is worthwhile to consider how domestic appliances have come to be anthropomorphized until now and what this might teach us about the commercial viability of the robotic vacuum cleaner. There are various robotic vacuum cleaners that are not anthropomorphized but are mobile and intelligent, in the sense of actually being capable of performing cleaning tasks independently of the user. These are now commercially well established, with dozens of different brands being sold worldwide. But the gesture robot is arguably more closely descended from a tendency in product design 
towards anthropomorphizing domestic items, which goes back much further.

The question of whether adding a face to an appliance, even one that is capable of gesturing, actually increases one's ability (or enjoyment) in using it is difficult to meaningfully engage with. There are clearly those who design based on this principle and Tokyo is filled with examples of cartoonish anthropomorphism in domestic product design and marketing. Appliances and other domestic items with faces can be seen in most homeware stores (Figure 3). I would argue that the prolific use of cartoonish faces in order to market household items is part of a broader kawaii (cute) aspect of the consumer culture of Japan. Sharon Kinsella defined the concept well in her writing on cute culture trends in the 1980's and 1990's: “Kawaii or 'cute' essentially means childlike; it celebrates sweet, adorable, innocent, pure, simple, genuine, gentle, vulnerable, weak, and inexperienced social behavior and physical appearances” (1995: p. $15)$. Her reference to kawaii as being that which may be considered childlike or simple is important in reading the kind of faces household items are given. Both in the examples of packaging and the gesture robot itself it is not an authentic human face, but a cartoon, an approximation that is being employed.

The kawaii aesthetic in consumer goods and its relationship with cartoons has been well explored. Botz-Bornstein decries the underlying ethos of the aesthetics of cuteness (kawairashisa):

“[kawaii] denotes a common popular culture closely linked to aesthetic expressions of kitsch, and which developed remarkably distinct features in all modernized East Asian countries. Modern East Asian popular culture bears traits of a social crisis that is most obvious in Japan, and which some people characterize as being 'all style and no substance.' A disillusionment with society as well as a psychological helplessness has created a youth culture that engages in consumption and the creation of a commodified dream world” (2008: p. 95).

Christine Yano's discussion of Japanese “cute-overload” explores the phenomenal success of Hello Kitty branded goods, which incidentally are also based on giving various items a friendly-face motif. In Yano's view "the interaction between viewer and viewed" is what defines the kawaii aesthetic (2009: p. 681). Brian McVeigh also used Hello Kitty and among other

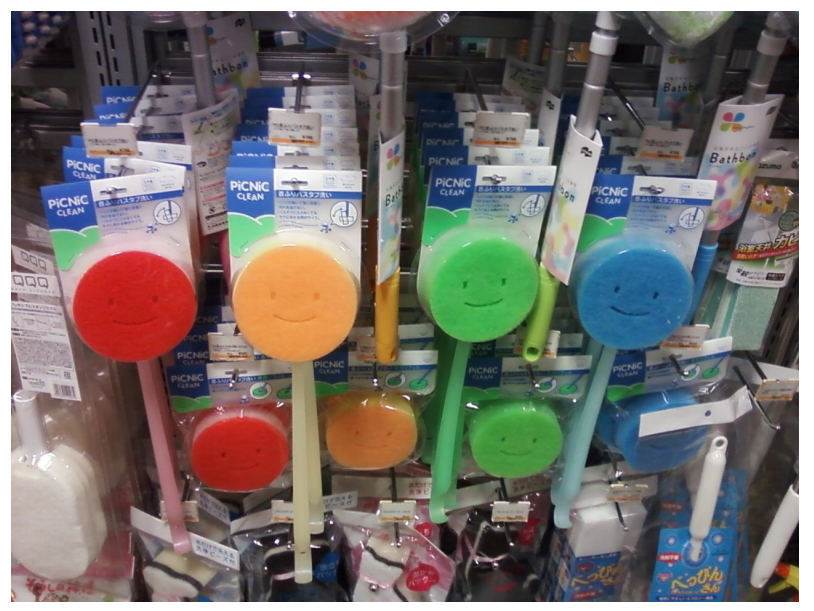

Figure 3.

Cleaning products with faces. brands in his discussion of "consumertopia," in order to explore how brands capitalize on the aesthetic relationship between cute, cool and camp (2000: p. 227). He refers to a "daily aesthetic of cuteness and campiness" defined as "an artificiality of manner or appearance, appreciated for its humor or triteness” (2000: p. 226). Again it is artificiality and novelty, which are central to achieving the desirable aesthetic. The addition of faces to domestic items in order to provoke a positive emotional response is an established practice and the gesture robot can arguably be seen as an extension of this principle. As with a mop-head that has a smiley face or all manner of domestic goods, which carry Hello Kitty and other popular character's faces, the purpose is, to evoke a positive emotional response (Figure 4). This, in Yano's terms, is to utilize the kawaii as sense of interaction between the viewer and the viewed.

While Japan may be the country where the design principle of cartoonish anthropomorphization of household objects is most easily seen, it is worth noting that the practice of giving domestic appliances humanlike attributes is certainly not unique to any one place. One non-Japanese example of this aesthetic in action is the British Somerset based homecare company Numatic International who market "Henry" the Hoover. Launched in 1980, there have been over seven million units sold, making it the company's flagship product and resulting in various spin off vacuum cleaners (Henrietta, Charles etc.). The company attributes the products success to its utility, keen to argue against the notion that it is its novelty appeal alone that has made it so successful. However the promotional material describes the vacuum cleaner in affectionate terms: " $A$ true professional in every sense of the word but never without that famous smile, loved and appreciated by users the world over... ask any Henry owner." The simple addition of a "cartoon mouth and eyes on their cylinder and a black top that could be mistaken for a bowler hat" makes the vacuum cleaner recognizable but also imbued with a sense of whimsy. The success of Henry vacuum cleaners demonstrates that the novel personification of a product may dramatically increase its appeal.

Returning to Japan, another common argument to support the necessity of using kawaii cartoonish designs for giving appliances faces relates to Mori Masahiro’s influential 1970 article “The Uncanny Valley” (Bukimi no Tani Genshō). The article drew on Freud's notion of the uncanny and applied it to robots



Figure 4.

Various products bearing the face of Rilakkuma. 
as well as all other manner of human facsimile. He argued that as human likenesses appear more realistic there is an increasing level of comfort and comfort up until a point. Surprisingly it is at the point when a human facsimile is almost fully convincing that it becomes most repellent to the observer. This sudden dip in familiarity is the "uncanny valley". Mori gives the example of prosthetic limbs to support this:

"Some prosthetic hands attempt to simulate veins, muscles, tendons, finger nails and finger prints, and their colour resembles human pigmentation. So maybe the prosthetic arm has achieved a degree of verisimilitude on a par with false teeth. But this kind of prosthetic hand is too real and when we notice it is prosthetic, we have a sense of strangeness... in this case the appearance is quite humanlike, but the familiarity is negative. This is the uncanny valley.” (1970: p. 34).

This theory is often employed to explain the potential causes of the ill-ease with which much science fiction approaches androids (Carpenter, 2006: p. 34). However it is also important in justifying the decision to make devices such as the gesture robot, and anthropomorphized appliances more generally, human in a way that is clearly novel and artificial rather than a more convincing way. It may be that keeping the faces and their range of expressions simplified and exaggerated actually makes it easier to evoke a positive emotional response. There have been psychological experiments conducted to investigate this principle, such as that of Robert Green and his colleagues. They recorded responses to images of people, androids and CGI characters with varying facial proportions and found that participant responses supported the idea that humanlike aesthetics could produce this uncanny effect. After the human facsimile becomes increasingly convincing, a point is eventually reached where it will become repellant (2008: p. 2470). There is clearly debate around the purpose and effect of anthropomorphizing appliances however for better or worse it has become an established aesthetic principle, which is particularly visible in Japanese domestic products. The vacuum gesture robot represents an extension of this idea in so much as it seeks not only to assist its user but to endear itself to them. The utilization of "emotional feedback," through the ability to create facial expressions and gestures in part owes itself to this earlier tradition of giving appliances faces.

The gesture robot has been designed not only to deliver spatial information but also to create facial expressions. Describing the experiment where older people were trained by the robot, Osawa gives an example of how emotions are used to emphasize the vacuum cleaner's statements: "when the voice says that 'vacuums can be difficult to maneuver,' our anthropomorphized system creates a sad expression” (2010: p. 182). I have witnessed examples of the gesture robot's happy and sad expressions. These are mainly achieved by changes in the brightness and size of the eyes together with arm gestures. This same experiment led Osawa and his colleagues to argue that a robot that gives emotional, and physical responses as well as simply information is more effective in assisting the elderly. I asked him recently about the importance of emotional feedback:

"Positive emotional feedback gives a feeling of acceptance and allowance toward users. (...) I estimate that periodical positive feedback is good for the learning process. Negative emotional feedback will arouse users senses and is understandable regardless attributes of each human (gender, age, culture). This intuitiveness will decrease cognitive load of the people in critical judgments.”

Emotional responses may assist in the retention of information being given by the gesture robot. Osawa went on to state that this kind of positive and negative feedback might be useful in disaster situations, where the user would be under stress and in need of considerable encouragement. In the experiment with elderly users it was also concluded that the emotive features of the robot simply evoke feelings of affability, that it is enjoyable for people to use (Osawa et al., 2010: p. 181). This aspect of the device, as with the aesthetics of cuteness should not be underestimated.

In order to achieve emotional responses from its users, empathy directed towards the anthropomorphic appliance is highly important. Bilge Mutlu conducted a study on the role of cooperation and competition in human-robot relations in which he found that people differ "in their perceptions of the robot and in their affective state based on whether they cooperate or compete with the robot” (2006: p. 2). The experiment was based on a game where participants were either paired with or competing against a robot. He argued, based on these findings, that people actually not only like the robot more, but actually find it more realistic when they cooperate with the device. In this case emotional feedback was crucial in shaping the relationship between the user and device. This relates to John Law's famous observation that machines can, depending on the circumstances, gain and lose humanlike attributes including intelligence, independence and even personal responsibility (1992: p. 4). An appliance such as the gesture vacuum robot that not only demonstrates an aesthetic anthropomorphism, but is literally able to scold or praise its user has this potential.

The feature of the vacuum gesture robot of which Osawa is most proud is its "imaginary body image". This is the term used to describe the device's ability to be aware of the user's position in relation to itself. This was achieved by using a module called "Body Image Creator" that uses marker detection to calculate precise positions through an algorithm of invert kinematics (2010: p. 181). Put simply it knows that if it is facing you, your left is its right. It also knows where its different parts are and how to direct you to them by gesturing, depending on your position in relation to it. It is this effect, which attracted my attention at the museum, and was most impressive in seeing the device in use. Osawa told me that he believes this is the best way to receive spatial information because unlike a map or even a virtual set of instructions on shown on a monitor the gesture robot is able to "share the space" of the user.

The Imaginary Body Image concept is arguably close to a form of proto-consciousness. It bears striking similarities to ideas in neuroscience concerning the role of mirror neurons. For instance in Thomas Metzinger's self-model theory of subjectivity (SMT) consciously experienced self and first person perspective emerges primarily through the development of the ability for introspective attention and motor skills. In selfmodels, there is no such thing as the substantial self as a distinct ontological entity but instead there is only "a dynamic, ongoing process creating very specific representational and functional properties" (2007: p. 4174). He also describes how the evolution of higher cognition generally can be described as an increasing ability to develop abstract forms of mental selfsimulation that will enable memory, learning and planning 
(2007: p. 4174). Under these terms the gesture robot approaches something close to consciousness. Also, in Metzinger's work on body image the concept of proprioception, how a sense of ownership is developed in relation to the body, is relevant to understanding how the gesture robot works. Body image and what is considered to be part of the body is continually altered and may be extended into the surrounding environment, for example through the use of tools: "neuro-scientific data indicate that any successful extension of behavioral space is mirrored in the neural substrate of the body image in the brain. The brain constructs an internalized image of the tool by assimilating it into the existing body image” (2009: p. 78). In this respect the gesture robot's ability to manipulate the user's, and its own, body image to a constructive end is a considerable achievement.

The gesture robot might also be viewed as the successful application of "active externalism" in thought. This is idea of Andy Clark and David J. Chalmers that the environment plays a crucial role in driving cognitive processes. They describe how an Alzheimer's patient with a piece of paper with directions to a certain address is equally able to find the address as someone with a good memory who has been told the route. For Clark and Chalmers these kinds of actions are not aids to thought but part of thought itself (1998: p. 14). In these terms the gesture robot would become not only a reflection or extension of the user's body image, but an active agent in their thought. The demonstration and description of how to use the vacuum cleaner allow the user potentially to think outside of themselves. Similarly, the importance of sensory experience over abstract thought alone is advocated by Edmund Carpenter in his discussion of total sensory experience (1972: p. 65). A benefit of the gesture robot's utilization of an imaginary body image is promoted by the claim that this can make it better at delivering spatial information. It is intended to assist in particular those whose capacity for abstract thought is in some respect limited, such as the elderly:

"having only auditory instructions sometimes causes misinterpretation of spatial information like locations and directions. This risk becomes large, especially with older people, because users' mental ability to manipulate images or patterns decreases with age. We support older people's learning of spatial information by using an attachable gesture robot. Our robot consists of human-like eyes and arms and is attached to the object. It supports older people's mental manipulation of space with gestures during voice interaction.” (2010: p. 179).

In my conversations with Osawa he often reiterated this potential application of the gesture robot. Having previously worked at MIT's Age lab and being acutely aware of elderly care as being the key topic currently in Japanese robotics funding and promotion, this is logical. Osawa was keen to add, however, that it may be equally appealing to a child or infant along similar argument based on impaired ability for abstract thought. The importance of pointing, gestures as part of "blended mental space" and how they reinforce learning are key considerations (2010: p. 184). The consistent theme in robotics of assisting with or compensating for the functional limitations of people is documented in Kathleen Richardson's work on androids at MIT. Where she recorded the irony that many of the androids she encountered during her research seemed to have been designed compensate for the designer's own physical limitations (2010: p. 83). For example, a robotic hand that was designed by an individual who had ongoing strain problems with his own hands that inhibited his ability to work. There are often references to the elderly and physical or mental impairment in robotics research where one is used as a euphemism for the other.

Osawa uses the term anthropomorphism generally as means of describing a sensation, "to feel social actors in the environment". The vacuum robot is designed to utilize the tendency in human thought to respond to gestures and spatial information as conveyed by other people. This is quite different to the humanlike qualities of empathy or cuteness as discussed earlier, but is at least as important. This confusion calls to mind Denis Vidal's argument, based his ethnography on the ritual interactions between humans and gods in the Western Himalayas, that we should consider anthropomorphism not as a single category distinction, but rather to see it as a system of various kinds of "sub-anthropomorphic" entities where human faculties can be gained and lost at will (2007: p. 925). The gesture vacuum robot employs several different kinds of human faculty (facial expressions, body image, language, emotional feedback) in order to become both figuratively and literally user-friendly.

At the museum where I carried out my fieldwork, there is a second exhibit, which employs the same kind of LED eye-like devices as are used for the gesture vacuum robot. It is a device art installation, "Nicodama” by Ryota Kuwakubo. Nicodama is the name of the robotic eyes that when lined up, start fluttering their eyelids and blinking. When attached to daily items they look as though they have acquired facial expressions. In this case a tea urn is transformed into a cute statue figure. Far less technologically sophisticated than the other devices I have been discussing but nevertheless simply through occasionally blinking and the general impression of a face, the urn becomes imbued with a personality. It is a playful contrast of traditional and contemporary Japanese design aesthetics, set significantly in a darkened "tearoom" enclosure within the exhibition space. Nicodama invites us to consider how easily a household item can be imbued with personality. It is transformed from inanimate to living through the simple addition of two lights that represent eyes. It speaks of the countless smiling faced objects in Japanese homeware stores as much as it does of the emerging technologies that make the eye movements possible. Quietly blinking in the darkened room, with the bodily proportions of a miniature snowman, it looks friendly. Anthropomorphism can be seen to be influential in the design of many domestic products and appliances in Japan as part of an aesthetic of cuteness. The vacuum gesture robot represents an extension of this principle as an appliance that is not only friendly in appearance, but is able also to engage in a kind of novel social interaction with its user, even to provoke an emotional response. The role of emotional feedback and the ability to invoke an awareness of its own body image heighten the potential for learning from the machine itself. In this respect the gesture vacuum robot is both figuratively and literally user-friendly.

\section{Summary}

It has been demonstrated how in various circumstances machines must be made to behave as human and people are made to behave as machines, as the friendly patrolling model of service staff behavior demonstrates. Osawa's vacuum gesture robot is a key example of the notion that devices work better 
when the interface appears to be human. Put simply a vacuum cleaner that can point at its own on button and tell you where it is might be easier to use than one that requires you study its instruction manual. There are also more superficial aesthetic advantages to giving a product a face as seen in the phenomenal success of various kawaii brands (Rilakkuma, Hello Kitty etc). The "friendly-patrolling" robot, with behaviour modeled on human security guards' non-threatening patterns of walking can also be seen as an expression of this tendency. The tendency towards the humanization of machines and mechanization of human behaviour is a relationship that has been critically commented on since the industrial revolution and continues to be influential in the development of new technology. In this manner the role of anthropomorphization can be seen in the aesthetic and functional design of consumer products in Japan.

\section{Acknowledgements}

I would like to thank Professor Michita Imai at Keio for introducing me to several of his researchers at his Information and Computer Science laboratory. Among those I met at Keio, I would like to thank Hirotaka Osawa for being so enthusiastic about my research and allowing me to interview him regarding his work. I am extremely grateful to the trustees for the Sutasoma Award, provided through the Royal Anthropological Institute to assist in cost of completing my doctoral thesis. I would also like to acknowledge the UCL Graduate School for providing financial support during my research.

\section{REFERENCES}

Bergson, H. (1956). Laughter. In Comedy (ed.), Wylie sypher (p. 72). New York: Garden City.

Botz-Bornstein, T. (2008). Wong Kar-wai's films and the culture of the kawaii. SubStance, 37, 94-109. http://dx.doi.org/10.1353/sub.0.0003

Carpenter, E. (1972). Oh, what a blow that phantom gave me! New York: Holt, Rinehart and Winston.

Carpenter, J., Eliot, M., \& Schulthe, D. (2006). The uncanny valley: Making human-nonhuman distinctions. Proceedings of the 5th International Conference on Cognitive Science, Vancouver: Canada Interaction.

Carroll, C. (2011). Us and them. National geographic.

http://ngm.nationalgeographic.com/2011/08/robots/carroll-text

Church, K., Weight, J., Berry, M., \& Macdonald, H. (2010). At home with media technology. Home Cultures, 7, 263-286.

http://dx.doi.org/10.2752/175174210X12785760502171

Clark, A., \& Chalmers, D. J. (1998). The extended mind. Analysis, 58, 10-23. http://dx.doi.org/10.1093/analys/58.1.7

Clarke, A. C. (1973). Profiles of the future. New York: Harper and Row.

Daniels, I. M. (2003). Scooping, raking, beckoning luck: Luck, agency and the interdependence of people and things in Japan. The Journal of the Royal Anthropological Institute, 9, 619-638.

http://dx.doi.org/10.1111/j.1467-9655.2003.00166.x

Gilson, M. (1998). A brief history of Japanese Robophilia. Leonardo, 31, 367-369. http://dx.doi.org/10.2307/1576597

Green, R., MacDorman, K. F., Ho, C., \& Vasudevan, S. (2008). Sensitivity to the proportions of faces that vary in human likeness. Com- puters in Human Behavior, 24, 2456-2474.

http://dx.doi.org/10.1016/j.chb.2008.02.019

Harkins, A. (1983). The computer, the robot and the sheep dog. Design Quarterly, 121, 22-29. http://dx.doi.org/10.2307/4091103

Hayashi, K., Shiomi, M., Kanda, T., \& Hagita, N. (2011). Friendly patrolling: A model of natural encounters. ATR Advanced Telecommunications Research Institute International. Kyoto, Japan.

Jack, I. (2010). Can manufacturing fill Britain’s economic vacuum? The Guardian. http://www.theguardian.com/commentisfree/2010/jul/24/can-manufact uring-fill-british-economic-vacuum

Kinsella, S. (1995). Cuties in Japan. In B. Moeran, \& L. Scov (Eds.), Women, media and consumption in Japan (pp. 220-255). Curzon: Hawaii University Press.

Law, J. (1992). Notes on the theory of actor network: Ordering, strategy and heterogeneity. Systems Practice, 5, 379-393. http://dx.doi.org/10.1007/BF01059830

McVeigh, B. J. (2000). How Hello Kitty commodifies the cute, cool and camp: "Consumutopia" versus "Control" in Japan. Journal of Material Culture, 5, 225-245. http://dx.doi.org/10.1177/135918350000500205

Metzinger, T. (2007). Self models. Scholarpedia, 2, 4174. http://dx.doi.org/10.4249/scholarpedia.4174

Metzinger, T. (2009). The ego tunnel: The science of the mind and the myth of the self. New York: Basic Books.

Mori, M. (1970). The uncanny valley. Energy, 7, 33-35.

Mutlu, B., Osman, S., Forlizzi, J., Hodgins, J., \& Kiesler, S. (2006). Perceptions of asimo: An exploration on co-operation and competition with humans vs humanoid robots. Human Robot Interaction, 2006, 351-352.

Occhi, D. J. (2012). Wobbly aesthetics, performance and message: Comparing Japanese kyara with their anthropomorphic forebears. Asian Ethnology, 71, 109-132.

Osawa, H., Ohmura, R., \& Imai, M. (2009). Using attachable humanoid parts for realizing imaginary intention and body image. International Journal of Social Robotics, 1, 109-123. http://dx.doi.org/10.1007/s12369-008-0004-0

Osawa, H., Orszulak, J., Godfrey, K. M., Imai, M., \& Coughlin, J. F. (2010). Improving voice interaction for older people using an attachable gesture robot. 19th IEEE International Symposium on Robot and Human Interactive Communication, September 2010. http://dx.doi.org/10.1109/ROMAN.2010.5598651

Richardson, K. (2010). Disabling as mimesis and alterity: Making Humanoid robots at the Massachusetts Institute of Technology. Enamor, Imitation, 22, 75-90.

Robertson, J. (2007). Robo sapiens Japanicus: Humanoid robots and the posthuman family. Critical Asian Studies, 39, 369-398. http://dx.doi.org/10.1080/14672710701527378

Sabelli, A. M., Kanda, T., \& Hagita, N. (2011). A conversational robot in an elderly care centre: An ethnographic study human robot. Interaction, 11 .

Vidal, D. (2007). Anthropomorphism or sub-anthropomorphism? An anthropological approach to gods and robots. Journal of the Royal Anthropological Institute, 13, 917-933. http://dx.doi.org/10.1111/j.1467-9655.2007.00464.x

Villiers de I'Isle-Adam, A. (2001). Tomorrow's eve. London: University of Illinois Press.

Webb, M. (1983). The robots are here! Design Quarterly, 121, 5.

Yano, C. R. (2009). Wink on pink: Interpreting Japanese cute as it grabs the global headlines. The Journal of Asian Studies, 68, 681688. http://dx.doi.org/10.1017/S0021911809990015 